\title{
AVALIAÇÃO DO USO DE ANTIDEPRESSIVOS E ANSIOLÍTICOS POR ACADÊMICOS DO CURSO DE ENFERMAGEM
}

\section{EVALUATION OF THE USE OF ANTIDEPRESSANTS AND ANXIOLYTICS BY STUDENTS OF THE NURSING COURSE}

\author{
Marla Brenda Pires Coimbra ${ }^{1}$ \\ Renata Aparecida Faria de Araujo ${ }^{1}$ \\ Patricia de Lima Lemos ${ }^{2}$ \\ Lorena Araujo Ribeiro ${ }^{1}$ \\ Helen Cristina Fávero Lisboa ${ }^{1}$
}

\begin{abstract}
Resumo: Pesquisas evidenciam sintomas depressivos e de ansiedade entre acadêmicos de enfermagem, que mediante expectativas, desafios e incertezas ao longo do curso, ainda convivem com condições estressantes relacionadas ao paciente, sendo frequente nesse momento, o início do uso de psicofármacos. 0 estudo teve como objetivo avaliar o uso de antidepressivos e ansiolíticos por acadêmicos do curso de Enfermagem. Trata-se de estudo transversal, descritivo e analítico, que utilizou questionário contendo informações sociodemográficas, econômicas e sobre uso de antidepressivos e ansiolíticos. Realizou-se o teste de qui-quadrado de Pearson para verificar associação entre as variáveis utiliza antidepressivos/ansiolíticos e sociodemográficas/econômicas. Foram entrevistados 79 alunos e destes, $13(16,4 \%)$ afirmaram fazer uso do medicamento. Em relação ao psicofármaco usado, os ansiolíticos foram os mais citados (52,63\%), prevalecendo a prescrição por psiquiatra $(46,15 \%)$, a maioria fazendo uso diário $(92,31 \%)$, iniciado a medicação após ingresso na Universidade $(61,54 \%)$ e nunca realizado alteração da dosagem sem consultar o médico (61,54\%). Um percentual de $76,92 \%$ assegurou ter conhecimento sobre os efeitos adversos e declararam saber que a remoção do medicamento deve ser feita de maneira gradual $(84,62 \%)$, no entanto $53,85 \%$ afirmaram ter interrompido o tratamento em algum momento sem consultar o médico. $\mathrm{Na}$ análise bivariada não houve associação entre as variáveis. Verificou-se a necessidade de ações voltadas para o acolhimento e escuta dos acadêmicos da área de enfermagem em relação aos transtornos depressivos e de ansiedade, e quanto ao uso racional e seguro dos psicofármacos, minimizando o sofrimento mental e as consequências do uso indevido dos fármacos.
\end{abstract}

Palavras-chave: Ansiolíticos. Antidepressivos. Saúde Mental. Curso de Enfermagem.

Abstract: Research shows evidence of depression and anxiety symptoms among nursing students, who, due to expectations, challenges and uncertainties throughout the course, still live with stressful conditions related to the patient, with frequent use of psychotropic drugs at this moment. The study aimed to evaluate the use of antidepressants and anxiolytics by students of the Nursing course. This is a crosssectional, descriptive and analytical study, which used a questionnaire containing sociodemographic and economic information, and information on the use of antidepressants and anxiolytics. Pearson's chi-square test was performed to verify the association between the variables of use of antidepressants / anxiolytics and the

\footnotetext{
${ }^{1}$ Curso de Enfermagem / Universidade Federal de Rondonópolis.

${ }^{2}$ Curso de Medicina / Universidade Federal de Rondonópolis.
} 
sociodemographic / economic variables. 79 students were interviewed, and 13 $(16.4 \%)$ of them said they used the medication. In relation to the psychotropic drug used, anxiolytics were the most cited $(52.63 \%)$, with the prescription by a psychiatrist $(46.15 \%)$ prevailing, the majority making daily use $(92.31 \%)$, starting the medication after entering University (61.54\%) and never changing the dosage without consulting the doctor $(61.54 \%)$. A percentage of $76.92 \%$ claimed they were aware of the adverse effects and stated that they knew the medication should be removed gradually (84.62\%); nonetheless, $53.85 \%$ affirmed they had stopped the treatment at some point without consulting the doctor. In the bivariate analysis, there was no association between the variables. There was a need for actions aimed at welcoming and listening to nursing students in relation to depressive and anxiety disorders, and regarding the rational and safe use of psychiatric drugs, minimizing mental suffering and the consequences of drug misuse.

Key words: Anxiolytics. Antidepressants. Mental Health. Nursing Course.

Data de submissão: 28.06 .2020

Data de aprovação: 30.11 .2020

Identificação e disponibilidade:

(https://revista.univap.br/index.php/revistaunivap/article/view/2516, http://dx.doi.org/10.18066/revistaunivap.v27i53.2516).

\section{INTRODUÇÃO}

Atualmente, os estudos epidemiológicos em saúde mental têm permitido maior compreensão da prevalência dos transtornos mentais e suas consequências no âmbito pessoal, familiar e social. Dessa forma, são estudados os transtornos mentais comuns (TMC), onde se incluem ansiedade e depressão, que irão expor um limiar entre saúde e doença, interferindo na qualidade de vida, com prejuízos e limitações, gerando um importante problema de saúde pública (BENER; TEWFIK, 2006).

A depressão se define como um transtorno de humor crônico caracterizado por sentimento de tristeza, perda de apetite, dificuldade de concentração, irritabilidade, indisposição entre outros sintomas, que ocasionam consequências negativas na vida do indivíduo e de seus familiares. E considerando o impacto social e aumento do número de casos, constitui um grave problema de saúde pública (RIBEIRO et al., 2014). A ansiedade se caracteriza por inquietação, distúrbios de sono, fadiga, dificuldade de concentração. Sintomas que incidem de forma negativa na vida do indivíduo, além de afetar a percepção motora e intelectual (FERNANDES et al., 2017). Ambos os transtornos, produzem morbidade desordenada bem como uso dos serviços de saúde (CHAVES et al., 2015).

A Organização das Nações Unidas (ONU) estima que a população mundial é composta por aproximadamente $30 \%$ de crianças e $14,2 \%$ adolescentes (ORGANIZAÇÃO..., 2012). O grupo de adolescente apresentando vulnerabilidade com elevadas taxas de mortalidade, onde umas das principais causas são as doenças psicoemocionais, que nos dias atuais começaram a aparecer com maior frequência entre os jovens, revelando altas taxas de prevalência de transtornos mentais nessa faixa etária. Estima-se que no mundo, um entre quatro a cinco jovens 
apresenta algum transtorno mental, e no Brasil uma prevalência de 7 a 12,7\% (THIENGO; CAVALCANTE; LOVISI, 2014).

Quando o cenário é o ambiente acadêmico, a situação torna-se ainda mais preocupante. Ainda que haja sentimentos positivos pelo ingresso na universidade, trata-se de uma fase de vulnerabilidades e de muitas expectativas, que por sua vez podem gerar situações de ansiedade em razão da insegurança e preocupações com o futuro, contribuindo para o surgimento do transtorno (PEUKER; FOGAÇA; BIZARRO, 2006). Nesta fase ocorrem diversas mudanças na vida dos estudantes, um período marcado por desafios e incertezas que pode ser a origem de problemas de saúde mental, como a ansiedade e depressão seguida dos comportamentos suicidários (GONÇALVES; FREITAS; SIQUEIRA, 2011).

Pesquisas brasileiras relatam que 15 a 29\% dos acadêmicos apresentam algum transtorno psíquico (SAKAE; PADÃO; JORNADA, 2010). Dentre os universitários, os da área da saúde merecem especial atenção, com maior nível de ansiedade, em relação aos demais (DEHGHAN-NAYERI; ADIB-HAJBAGHERY, 2011). Estudantes dessa área, incluindo os de enfermagem, vivenciam durante a graduação situações que geram ansiedade (MARCHI et al., 2013). Estas situações podem ser desencadeadas por diversos fatores como: a rotina na prática clínica, o convívio com o sofrimento humano, a presença dos tutores no cenário da prática, além da condição altamente estressante do processo de morte e morrer de um paciente (MELINCAVAGE, 2011; LIMA; NIETSCHE; TEIXEIRA, 2012).

Diante desse cenário, ocorre a busca por estratégias que visem essa adaptação, incluindo a procura por ajuda, sendo frequente nesse momento o início do uso de psicofármacos, o que muitas vezes ocorre de forma indevida (PICOLOTTO et al., 2010). Nesse contexto, considerando o aumento da prevalência de transtornos mentais entre os estudantes universitários e as consequências do tratamento inadequado, o trabalho realizado teve como objetivo avaliar o perfil e uso de antidepressivos e ansiolíticos por acadêmicos do curso de Enfermagem.

\section{METODOLOGIA}

Trata-se de estudo transversal, com componente descritivo e analítico. A coleta de dados aconteceu no período de agosto de 2019 a fevereiro de 2020, por meio de entrevista, utilizando-se um questionário elaborado com perguntas objetivas que faziam referência a dados de identificação sociodemográficos, uso de antidepressivos e ansiolíticos e a assistência prestada pelas equipes de saúde. As entrevistas foram realizadas individualmente nas dependências da Universidade Federal de Rondonópolis, em local reservado para garantir a privacidade do participante.

A amostra foi selecionada intencionalmente conforme disponibilidade do acadêmico no local da pesquisa. A população do estudo foi constituída por acadêmicos do curso de Graduação em Enfermagem com idade igual ou superior a 18 anos, que estavam regularmente matriculados no curso e que aceitaram participar da pesquisa e assinaram o Termo de Consentimento Livre e Esclarecido. Estes foram abordados pessoalmente em seu local de estudo, onde lhes foi apresentado os objetivos da pesquisa, riscos e benefícios de sua participação e garantia de sigilo durante toda a pesquisa, inclusive na divulgação da mesma. Foram excluídos da pesquisa os alunos que não possuem vínculo com a UFR/CUR; pós-graduandos; alunos ouvintes e especiais e os pesquisadores envolvidos no 
projeto.

A coleta de dados ocorreu no período matutino e vespertino de acordo com o horário de funcionamento do curso. Os dados obtidos foram digitados em planilha digital e os resultados apresentados em tabelas, utilizando frequências relativa e absoluta. A análise de associação, entre a variável utiliza medicamento antidepressivo e ansiolítico (desfecho) e caraterísticas sociodemográficas e econômicas, foi realizada com teste de qui-quadrado de Pearson no programa estatístico Stata versão 12.0 (StataCorp LP, College Station®, Estados Unidos da América do Norte). Adotou-se nível de significância de 5\%.

A pesquisa foi pautada na Resolução 466/2012 (BRASIL, 2012) que dispõe sobre diretrizes e normas regulamentadoras de pesquisas envolvendo seres humanos do Conselho Nacional de Saúde (CNS). As entrevistas foram iniciadas após a aprovação do projeto pelo Comitê de Ética em pesquisa (CAAE: 96080718.0.0000.8088).

\section{RESULTADOS E DISCUSSÃO}

Do total de estudantes entrevistados ( $n=79), 13$ (16\%) utilizam ansiolíticos e/ou antidepressivos, um resultado semelhante ao encontrado por Istilli et al. (2010), que relata em seu trabalho $19 \%$ dos estudantes de enfermagem utilizando tal classe de medicamentos. Outro estudo realizado em um curso de Graduação em Enfermagem no Estado de Minas Gerais evidenciou que $26 \%$ dos 72 alunos entrevistados eram usuários de antidepressivos, a maioria do sexo feminino (79\%) (TELES FILHO; PEREIRA JUNIOR, 2013).

A amostra dessa pesquisa é composta em sua maioria por pessoas do sexo feminino $(93,67 \%)$, solteiras $(81,01 \%)$, sem filhos $(88,61 \%)$, que não exercem atividade profissional remunerada $(63,29 \%)$ e que residem com a família $(83,54 \%)$. Entre os entrevistados, prevaleceu a renda familiar entre 2 a 3 salários mínimos $(29,11 \%)$ e indivíduos que referiram não dispor de plano de saúde $(50,63 \%)$. À análise bivariada, não se observou associação entre as variáveis: utilização de medicamento ansiolítico e antidepressivo e características sociodegráficas e econômicas (Tabela 1).

Tabela1 - Análise bivariada e características sociodemográficas e econômicas dos acadêmicos de enfermagem da Universidade Federal de Rondonópolis, 2019-2020

\begin{tabular}{|c|c|c|c|c|}
\hline \multirow{2}{*}{ Variáveis } & \multirow{2}{*}{$\mathrm{n}(\%)$} & \multicolumn{2}{|c|}{ Uso de Medicamento } & \multirow{2}{*}{ p-valor* } \\
\hline & & Sim & Não & \\
\hline \multicolumn{5}{|l|}{ Sexo } \\
\hline Masculino & $5(6,33)$ & $11(14,86)$ & $63(85,14)$ & \multirow{2}{*}{0,142} \\
\hline Feminino & $74(93,67)$ & $2(40,00)$ & $3(60,00)$ & \\
\hline \multicolumn{5}{|l|}{ Estado Civil } \\
\hline Solteiro & $64(81,01)$ & $9(14,06)$ & $55(85,94)$ & \multirow{2}{*}{0,236} \\
\hline Casado & $15(18,99)$ & $4(26,67)$ & $11(73,33)$ & \\
\hline \multicolumn{5}{|l|}{ Filhos } \\
\hline Não possui & $70(88,61)$ & $10(14,29)$ & $60(85,71)$ & \multirow{2}{*}{0,147} \\
\hline Possui & $9(11,39)$ & $3(33,33)$ & $6(66,67)$ & \\
\hline
\end{tabular}


Tabela1 - Análise bivariada e características sociodemográficas e econômicas dos acadêmicos de enfermagem da Universidade Federal de Rondonópolis, 2019-2020

(conclusão)

\section{Trabalha}

Sim

Não

$\begin{array}{lll}29(36,71) & 4(13,79) & 25(86,21) \\ 50(63,29) & 9(22,50) & 31(77,50)\end{array}$

Possui plano de saúde

Sim

$39(49,37) \quad 6(15,38)$

$33(84,62)$

0,800

Não

$40(50,63) \quad 7(17,50) \quad 33(82,50)$

\section{Morador que reside}

Família

Amigos

$66(83,54) \quad 10(15,15)$

$56(84,85)$

$2(2,53) \quad 1(50,00)$

$1(50,00)$

0,418

Sozinho

$11(13,92)$

$2(18,18)$

$9(81,82)$

Renda familiar em salários mínimos*

Até 1

Entre 1 e 2

$8(10,13)$

$2(25,00)$

$6(75,00)$

Entre 2 e 3

$21(26,58)$

$4(19,05)$

23(29,11)

$2(8,70)$

$17(80,95)$

$21(91,30)$

0,510

Entre 3 e 4

$11(13,92) \quad 3(27,27)$

$8(72,73)$

* Teste do Qui-quadro de Pearson.

Fonte: Elaborado pelos autores.

O maior percentual de mulheres observado em nosso estudo, pode estar relacionado às características do curso de enfermagem, composto em sua maioria, por acadêmicos do sexo feminino. As aulas em período integral e o tempo ocupado pelas atividades acadêmicas diminui a possibilidade de o estudante trabalhar, podendo acarretar dificuldades financeiras que geram outros problemas como a redução da capacidade de raciocínio e redução do desempenho escolar com consequente sentimento depressivo expresso pela tristeza, culpa e impotência (AMADUCCI; MOTA; PIMENTA, 2010). Além de todos esses agravantes, a baixa renda das famílias, pode desencadear preocupações exageradas no estudante, o que também pode interferir no seu desempenho (TELES FILHO; PEREIRA JUNIOR, 2013).

Quando os usuários de antidepressivos e/ou ansiolíticos foram questionados sobre qual psicofármaco em uso, muitos alegaram não lembrar o nome, no entanto citaram a classe do medicamento, e alguns dos entrevistados demonstraram que faziam uso de mais de um medicamento. Dessa forma, os fármacos foram agrupados por classes sendo citados os ansiolíticos (52,63\%), antidepressivos $(21,05 \%)$, antipsicóticos $(10,52 \%)$, estabilizadores de humor (10,52\%) e fitoterápicos $(5,26 \%)$.

A ansiedade foi a principal indicação para uso do medicamento $(61,54 \%)$ seguida pela depressão (30,77\%). Quanto ao profissional responsável pela prescrição, 46,15\% referiu obtê-la mediante indicação realizada por psiquiatra. Um percentual de $92,31 \%$ faz o uso diário, tendo adquirido a medicação pela farmácia privada $(92,31 \%)$ e iniciado o tratamento após ingresso na Universidade $(61,54 \%)$. A 
maioria utiliza a medicação de 1 a 5 anos (38,46\%), e não faz uso de outro medicamento além do psicofármaco citado (69,23\%) (Tabela 2).

Tabela 2 - Distribuição da utilização de antidepressivos/ansiolítico por acadêmicos de enfermagem da Universidade Federal de Rondonópolis, 2019-2020.

\begin{tabular}{lcc}
\hline \multicolumn{1}{c}{ Variáveis } & $\mathbf{n}$ & $\mathbf{( \% )}$ \\
\hline $\begin{array}{l}\text { Qual foi a indicação para o uso? } \\
\quad \text { Ansiedade }\end{array}$ & 8 & 61,54 \\
$\quad$ Depressão & 4 & 30,77 \\
$\quad$ Outra & 1 & 7,69 \\
Quem prescreveu: & 6 & 46,15 \\
$\quad$ Psiquiatra & 3 & 23,08 \\
$\quad$ Clínico geral & 1 & 7,69 \\
$\quad$ Neurologista & 3 & 23,08 \\
$\quad$ Outro & & \\
Uso & 12 & 92,31 \\
$\quad$ Diário & 1 & 7,69 \\
$\quad$ Esporadicamente quando acha necessário & & \\
Quando começou a usar & 5 & 38,46 \\
$\quad$ Antes do ingresso na Universidade & 8 & 61,54 \\
$\quad$ Após o ingresso na Universidade & & \\
Onde adquiri o medicamento & 1 & 7,69 \\
$\quad$ Farmácia da rede pública & 12 & 92,31 \\
$\quad$ Farmácia Privada & & \\
Tempo de Uso & 3 & 23,08 \\
$\quad$ Menos de 6 meses & 5 & 38,46 \\
$\quad$ 6 meses a 1 ano & & 38,46 \\
$\quad$ I a 5 anos \\
Faz uso de outro medicamento além do \\
ansiolítico/antidepressivo \\
$\quad$ Não \\
$\quad$ Sim
\end{tabular}

Fonte: Elaborado pelos autores.

Um elevado percentual $(61,54 \%)$ relatou fazer uso de psicofármaco por motivo de ansiedade. Estudos mostram que entre estudantes de enfermagem, é comum o uso de benzodiazepínicos, não se considerando os danos decorrentes de seu uso como os prejuízos nas funções cognitivas e psicomotoras relacionados à administração prolongada da droga, independente da dose (PICOLOTTO et al., 2010). Estes efeitos estão associados à depressão do sistema nervoso central (SNC) causada pelo fármaco que pode ter como consequência o prejuízo na memória, diminuição da atividade psicomotora, além da possibilidade de causar dependência, tolerância, potencialização do efeito depressor quando em uso concomitante com o álcool e outras drogas depressoras (ORTEGA-PÉREZ; COSTAJÚNIOR; VASTERS, 2011).

De modo geral, jovens acadêmicos estão mais vulneráveis a alterações emocionais em decorrência de condição social a que estão sujeitos. Apesar de preparados biologicamente, vivenciam a transformação psicológica, podendo ter imaturidade para assumir o novo ritmo de vida imposta pela jornada acadêmica, sobrecarga de estudos, distanciamento da família, alimentação irregular, entre outros (EISENBERG et al., 2011). 
O sofrimento mental, relacionado à ansiedade e depressão, tem sido destaque nas últimas décadas, provavelmente em virtude de maior número de diagnósticos e aumento da disponibilidade de novos fármacos (ROCHA; WERLANG, 2013). Pesquisa realizada em uma universidade pública de Recife encontrou a maior parte dos acadêmicos em uso de ansiolíticos (31,3\%) (AQUINO; BARROS; SILVA, 2010) concordante com o presente estudo em que a maior parte dos acadêmicos entrevistados relatou fazer uso de ansiolíticos $(52,63 \%)$ dados semelhantes em pesquisa realizada em uma escola de Enfermagem em São Paulo, que encontrou $19 \%$ dos alunos em uso de antidepressivos, onde $21,05 \%$ dos estudantes do curso de enfermagem faziam uso da mesma classe de medicamento (ISTILLI et al., 2010).

A utilização de psicofármacos muitas vezes é realizada de forma irracional, fato que ocorre por diferentes motivos entre eles a automedicação, erro de prescrições e aumento de enfermidades relacionadas a transtornos mentais (ORLANDI; NOTO, 2005). É importante ressaltar que o uso crônico dessas substâncias pode resultar na dependência química (GRUBER; MAZON, 2014), e a abstinência vem a ser um importante fator relacionado a severo prejuízo à vida social, considerando sintomas como à irritabilidade, à insônia excessiva, sudorese, dores no corpo a até convulsões (CARLINI et al., 2001). Dessa forma, o uso indevido de ansiolíticos e/ou antidepressivos pode causar impactos negativos no âmbito econômico e social quando relacionados aos prejuízos no aprendizado e dificuldades nas relações familiares, além do aumento de gastos na saúde pública com tratamento de dependentes químicos por gerar problemas associados à intoxicação (LOPES; GRIGOLETO, 2011).

Quanto ao conhecimento dos entrevistados acerca do psicofármaco em uso, $61,54 \%$ afirmaram não terem alterado a dosagem sem consultar o médico e $76,92 \%$ asseguraram conhecimento sobre os efeitos adversos do medicamento em uso. Quanto aos ansiolíticos/antidepressivos 76,92\% referiram acreditar que tais medicamentos podem causar dependência, tolerância. Dos participantes, 84,62\% dizem saber que o medicamento, ao final do tratamento, deve ser removido de maneira gradual e $53,85 \%$ declararam ter interrompido o tratamento em algum momento sem consultar o médico. Sobre dúvidas ao tratamento ou medicamento, $69,23 \%$ afirmou não possuir (Tabela 3 ).

Em estudo realizado por Marchi et al. (2013), foi constatado que $27 \%$ e $46 \%$ dos acadêmicos aumentaram a dose do medicamento e interromperam o tratamento sem consultar o médico, respectivamente. Na atual pesquisa foi encontrado um percentual de $38,46 \%$ dos estudantes que já aumentaram ou diminuíram a dose do medicamento sem consultar o médico e 53,85\% já interrompeu o tratamento sem orientação médica, ainda que a maioria tenha respondido afirmar saber que a remoção do medicamento deve ser de maneira gradual $(84,62)$. Diante dos dados citados, é necessário destacar os riscos inerentes ao aumento ou diminuição das doses sem indicação médica, lembrando que esta deve ser feita com orientação do profissional para redução quando necessária de forma gradativa e sem riscos à saúde. 
Tabela 3 - Distribuição dos estudantes de enfermagem de acordo com o conhecimento sobre o ansiolítico/antidepressivo em uso. Universidade Federal de Rondonópolis, 2019-

\begin{tabular}{|c|c|c|c|c|c|c|}
\hline \multirow{2}{*}{ QUESTÕES } & \multicolumn{2}{|c|}{ SIM } & \multicolumn{2}{|c|}{ NÃO } & \multicolumn{2}{|c|}{ NÃO SABE } \\
\hline & $(\%)$ & $\mathbf{n}$ & $(\%)$ & $\mathbf{n}$ & $(\%)$ & $\mathbf{n}$ \\
\hline $\begin{array}{l}\text { Já aumentou ou diminuiu a dose sem } \\
\text { consultar o médico? }\end{array}$ & 38,46 & 5 & 61,54 & 8 & - & - \\
\hline $\begin{array}{l}\text { Conhece as ações e os efeitos } \\
\text { adversos do medicamento que utiliza? }\end{array}$ & 76,92 & 10 & 23,08 & 3 & - & 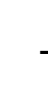 \\
\hline $\begin{array}{l}\text { Os ansiolíticos/antidepressivos podem } \\
\text { causar dependência? }\end{array}$ & 76,92 & 10 & - & - & 23,08 & 3 \\
\hline $\begin{array}{l}\text { Os ansiolíticos/antidepressivos podem } \\
\text { causar tolerância? }\end{array}$ & 76,92 & 10 & - & - & 23,08 & 3 \\
\hline $\begin{array}{l}\text { A remoção do medicamento no final } \\
\text { do tratamento deve ser feita de } \\
\text { maneira gradual? }\end{array}$ & 84,62 & 11 & - & - & 15,38 & 2 \\
\hline $\begin{array}{l}\text { Em algum momento você interrompeu } \\
\text { o tratamento sem consultar o médico? }\end{array}$ & 53,85 & 7 & 46,15 & 6 & - & - \\
\hline $\begin{array}{l}\text { Tem dúvidas sobre o tratamento ou } \\
\text { sobre o medicamento? }\end{array}$ & 30,77 & 4 & 69,23 & 9 & - & - \\
\hline
\end{tabular}

Fonte: Elaborado pelos autores

Os participantes consideraram importante a orientação sobre tratamento, no entanto nem todos foram orientados (Tabela 4). Marchi et al. (2013) destacaram em sua pesquisa, que $43,8 \%$ dos acadêmicos possuíam dúvidas quanto ao tratamento, um resultado semelhante ao encontrado em nossa pesquisa $(30,77 \%)$. Muitas vezes a presença de dúvidas pode estar relacionada à má conduta de interromper o tratamento ou aumentar a dose do fármaco sem indicação médica. Faz-se, necessário a execução de um plano assistencial que contemple ações de educação em saúde, com vistas a esclarecer dúvidas a respeito do psicofármaco, alertando quanto aos possíveis riscos e danos à saúde.

Pesquisa destaca hegemonia do modelo biomédico que abrevia o tempo para questionamentos sobre a proposta terapêutica e a falta de iniciativa do paciente em questionar os profissionais sobre a medicação em uso (RIBEIRO et al., 2014). Apesar de um expressivo número de acadêmicos $(46,15 \%)$ não achar importante os serviços das Estratégia Saúde da Família (ESF) para o tratamento, ressalta-se que as ESFs constituem um campo oportuno para o desenvolvimento de ações voltadas à saúde mental, mantendo a universalidade e integralidade, do atendimento para com o usuário. 
Tabela 4 - Distribuição dos estudantes de enfermagem, usuários de antidepressivos/ansiolíticos, de acordo com a opinião sobre a importância da orientação no tratamento. Universidade Federal de Rondonópolis/2019-2020.

\begin{tabular}{lcc}
\multicolumn{1}{c}{ Variáveis } & $\mathbf{n}$ & $\%$ \\
\hline $\begin{array}{l}\text { Considera a orientação sobre o tratamento } \\
\text { importante? }\end{array}$ & \\
$\quad$ Sim & 13 & 100 \\
Não & 0 & 0 \\
$\quad$ Não sabe & 0 & 0 \\
Você recebeu essa orientação? & 9 & \\
$\quad$ Sim & 4 & 69,23 \\
$\quad$ Não & & 30,77 \\
Qual o profissional da saúde o fez? & 10 & 76,92 \\
$\quad$ Médico prescritor & 1 & 7,69 \\
$\quad$ Farmacêutico & 1 & 7,69 \\
$\quad$ Enfermeiro & 1 & 7,69 \\
$\quad$ Outro profissional & & \\
Em que aspecto a orientação interfere? & 6 & 46,15 \\
$\quad$ Aumento da confiança na terapia & 2 & 15,38 \\
$\quad$ Aumento da segurança na terapia & 2 & 15,38 \\
$\quad$ Aumento da efetividade na terapia & 1 & 7,69 \\
$\quad$ Reduz efeitos indesejados & 2 & 15,38 \\
$\quad$ Reduz interação com outras drogas & 4 & 30,77 \\
$\quad$ Todos & & \\
Você considera importante os serviços de saúde da & & 53,85 \\
sua ESF para o seu tratamento? & 7 & 46,15 \\
$\quad$ Sim & 6 & \\
Não & &
\end{tabular}

Fonte: Elaborado pelos autores

No estudo realizado por Cunha et al. (2009), ressalta a contribuição do professor para criar vínculo com o estudante fortalecendo o processo de orientação, podendo contribuir para o aumento da confiança, segurança e conhecimento sobre tratamento medicamentoso. Dessa maneira destaca-se também, o papel de toda a equipe frente aos usuários de antidepressivos e/ou ansiolíticos. De forma semelhante, outro estudo, descreve que para os estudantes entrevistados, a equipe de saúde e o professor podem ser os primeiros a atuar na detecção e manejo dos casos de transtornos dessa natureza (FUREGATO; SANTOS; SILVA, 2008). É essencial que o conhecimento desses profissionais seja usado para orientar seus acadêmicos de forma segura e adequada, reforçando o uso racional e consciente.

Por fim, é fundamental o estudo das relações entre transtornos mentais, atenção e desempenho acadêmico, considerando-se que as dificuldades apontadas como a falta de informação, percepção e apoio por parte dos professores e familiares acerca da depressão/ansiedade nos acadêmicos pode contribuir para o aumento das dificuldades enfrentadas.

\section{CONCLUSÃO}

Considerando que a amostra deste estudo é composta por estudantes de enfermagem, é relevante a informação da existência de um percentual acadêmico que ainda possui dúvidas quanto ao uso de antidepressivos e ansiolíticos. Este dado 
é preocupante, uma vez que se acredita que os acadêmicos de enfermagem são detentores de conhecimento necessário para orientar os pacientes a respeito do uso racional, riscos e benefícios dessa classe farmacológica.

Os resultados encontrados no presente estudo apontam para a necessidade de ações voltadas para a valorização do acadêmico de enfermagem e outras áreas da saúde com adoção, pela instituição formadora, de medidas direcionadas aos fatores de risco para sofrimentos mentais entre os estudantes, propondo estratégias que viabilizem a escuta e acolhimento visando minimizar suas consequências.

Nesse contexto, é relevante destacar a necessidade de parceria entre instituição universitária e profissionais da Estratégia Saúde da Família (ESF), Centros de Atenção Psicossocial (CAPS), Núcleos de Apoio à Saúde da Família (NASF) entre outros, tendo como objetivo desenvolver projetos que promovam encontros para discussões, avaliações, acompanhamento e orientações aos acadêmicos em sofrimento mental. Tais ações devem buscar reduzir o uso precoce e inadequado de psicofármacos, além de estimular hábitos de vida saudáveis que irão contribuir para melhor qualidade de vida e consequentemente uma boa saúde mental. Além disso, se faz importante que a instituição acadêmica também ofereça aos professores capacitação adequada para detectarem sinais de sofrimento mental entre os estudantes, contribuindo com os docentes na tomada de decisões e abordagens aos indivíduos em situação de vulnerabilidade.

No que concerne às limitações do estudo, ressalta-se que não foi possível realizar análise múltipla devido ao número limitado de participantes, com isso recomenda-se estudos adicionais para explorar a temática.

\section{REFERÊNCIAS}

AMADUCCI, C. M.; MOTA, D. D. F. C.; PIMENTA, C. A. M. Fadiga entre estudantes de graduação em enfermagem. Revista da Escola de Enfermagem, São Paulo, v. 44, n. 4, p. 1052-1058, 2010.

AQUINO, D. S.; BARROS, J. A. C.; SILVA, M. D. P. A automedicação e os acadêmicos da área de saúde. Ciência \& Saúde Coletiva, Rio de Janeiro, v. 15, n. 5, p. 2533-2538, 2010.

BENER, A.; TEWFIK, I. Prevalence of overweight, obesity, and associated psychological problems in Qatari's female population. Obesity Reviews, v. 7, p. 139145, 2006.

BRASIL. Ministério da Saúde. Resolução oㅜ 466, de 12 de dezembro de 2012.

Diário Oficial da União, Brasília, 2012. Disponível em: https://bvsms.saude.gov.br/bvs/saudelegis/cns/2013/res0466_12_12_2012.html, Acesso em: 2 set. 2020

CARLINI, E. A. et al. Drogas psicotrópicas: o que são e como agem. Revista Imesc, v. 3, p. 9-35, 2001.

CHAVES, E. C. L. et al. Anxiety and spirituality in university students: a crosssectional study. Revista Brasileira de Enfermagem, Brasília, v. 68, n. 3, p. 444-9, 2015. 
CUNHA, M. A. B. et al. Transtornos psiquiátricos menores e procura por cuidados em estudantes de Medicina. Revista Brasileira de Educação Médica, Rio de Janeiro, v. 33, n. 3, p. 321-328, set. 2009.

DEHGHAN-NAYERI, N.; ADIB-HAJBAGHERY, M. Effects of progressive relaxation on anxiety and quality of life in female students: A non-randomized controlled trial. Alternat Complement Ther, v. 19, n. 4, p.194-200, 2011.

EISENBERG, D. et al. Mental health service utilization among college students in the United States. J Nerv Ment Dis, v. 199, n. 5, p. 301-8, 2011.

FERNANDES, M. A. et al. Transtornos de ansiedade: vivências de usuários de um ambulatório especializado em saúde mental. Revista de Enfermagem UFPE on line, Recife, v. 11, n. 10, p. 3836-44, out. 2017.

FUREGATO, A. R. F.; SANTOS, J. L. F.; SILVA, E. C. Depression among nursing students associated to their self-esteem, health perception and interest in mental health. Revista Latino-americana de Enfermagem, Ribeirão Preto, v. 16, n. 2, p.198-204, 2008.

GONÇALVES, A.; FREITAS, P.; SEQUEIRA, C. Comportamentos Suicidários em Estudantes do Ensino Superior: Factores de Risco e de Protecção. Millenium, v. 40, p. 149-159, nov. 2011.

GRUBER, J.; MAZON, L. M. A prevalência na utilização de medicamentos psicotrópicos no município de Mafra: um estudo retrospectivo. Saúde e Meio Ambiente: Revista Interdisciplinar, v. 3, n.1, p. 44-50, jul. 2014.

ISTILLI, P. T. et al. Antidepressivos: uso e conhecimento entre estudantes de enfermagem. Revista Latino-Americano de Enfermagem, Rio de Janeiro, v. 18, n. 3, p.131-139, jun. 2010.

LIMA, M. G. R.; NIETSCHE, E. A.; TEIXEIRA, J. A. Reflexos da formação acadêmica na percepção do morrer e da morte por enfermeiros. Revista Eletrônica de Enfermagem, v. 14, n. 1, p.181-188, jan./mar. 2012.

LOPES, L. M. B.; GRIGOLETO, A. R. L. Uso consciente de psicotrópicos: responsabilidade dos profissionais da saúde. Brazilian Journal of Health, v. 2, n.1, p. 1-14, jan./abr. 2011.

MARCHI, K. C. et al. Ansiedade e consumo de ansiolíticos entre estudantes de enfermagem de uma universidade pública. Revista Eletrônica de Enfermagem, v. 15, n. 3, p. 731-739, jul./set. 2013.

MELINCAVAGE, S. M. Student nurses' experiences of anxiety in the clinical setting. Nurse Educ Today, v. 31, n. 8, p. 785-789, 2011.

ORGANIZAÇÃO DAS NAÇÕES UNIDAS (ONU). Objetivos de Desenvolvimento do Milênio: Mapa do Progresso de 2012. Nova York: Divisão de Estatística do Departamento de Assuntos Econômicos e Sociais; 2012. 
ORLANDI, P.; NOTO, A. R. Uso indevido de benzodiazepínicos: um estudo com informantes-chave no município de São Paulo. Revista Latino-americana de Enfermagem, Ribeirão Preto, v. 13, p. 896-902, out. 2005.

ORTEGA-PÉREZ, C. A.; COSTA-JÚNIOR, M. L.; VASTERS, G. P. Perfil epidemiológico de la drogadicción en estudiantes universitários. Revista LatinoAmericana de Enfermagem, Ribeirão Preto, v. 19, p. 665-672, jun. 2011.

PEUKER, A. C.; FOGAÇA, J.; BIZARRO, L. Expectativas e beber problemático entre universitários. Psicologia: Teoria e Pesquisa, Brasília, v. 22, n. 2, p. 193-200, 2006.

PICOLOTTO, E. et al. Prevalence and factor associated with psychoactives substances consumption for academics of Nursing of the University of Passo Fundo. Ciência e Saúde Coletiva, Rio de Janeiro, v. 15, n. 3, p. 645-654, 2010.

RIBEIRO, A. G. et al. Antidepressivos: uso, adesão e conhecimento entre estudantes de medicina. Revista Ciência e Saúde Coletiva, Rio de Janeiro, v. 19, n. 6, p. 18251833, 2014.

ROCHA, B. S.; WERLANG, M. C. Psicofármacos na Estratégia Saúde da Família: perfil de utilização, acesso e estratégias para a promoção do uso racional. Ciência e Saúde Coletiva, Rio de Janeiro, v. 18, n. 11, p. 3291-3300, nov. 2013.

SAKAE, T. M.; PADÃO, D. L.; JORNADA, L. K. Sintomas depressivos em estudantes da área da saúde em uma Universidade no Sul de Santa Catarina - UNISUL. Revista AMRIGS, v. 54, n. 1, p. 38-43, mai./jun. 2010.

TELES FILHO, P. C. P. T.; PEREIRA JUNIOR, A. D. C. P. Antidepressivos: consumo, orientação e conhecimento entre acadêmicos de enfermagem. Revista de Enfermagem do Centro Oeste Mineiro, v. 3, n. 3, p. 829-836, set./dez. 2013.

THIENGO, D. L.; CAVALCANTE, M. T.; LOVISI, G.M. Prevalência de transtornos mentais entre crianças e adolescentes e fatores associados: uma revisão sistemática. J Bras Psiquiatr. Rio de Janeiro, v. 63, n. 4, p. 360-372, out./dez. 2014. 